\title{
Impacto das políticas de tratamento dos excessos de estoque sobre o efeito chicote em ambientes com demanda estocástica
}

\author{
José Carlos Fioriolli ${ }^{1}$; Flávio Sanson Fogliatto ${ }^{2}$
}

\begin{abstract}
Resumo: A amplificação da variabilidade da demanda ao longo de uma cadeia de abastecimento é um fenômeno conhecido como Efeito Chicote (EC). Os principais modelos de quantificação deste fenômeno disponíveis na literatura utilizam o mesmo pressuposto no cálculo do tamanho dos pedidos: eventuais valores negativos correspondem a excessos de estoque que podem (i) ser devolvidos sem custo; ou (ii) ser totalmente desconsiderados. De acordo com os autores desses modelos, o modo como os excessos são tratados não produz impactos relevantes sobre a intensidade do EC. Diferentemente, neste artigo demonstra-se que a escolha de uma política adequada de tratamento dos excessos de estoque é fundamental para uma quantificação mais precisa do EC. Propõe-se, assim, uma nova política para tratamento desses excessos, capaz de evitar a amplificação desnecessária da variabilidade produzida por políticas inadequadas sobre os níveis de demanda.
\end{abstract}

\begin{abstract}
The amplification in demand variability throughout the elements of a supply chain is known as the Bullwhip Effect (BE). The best known models available in the literature to quantify the BE are based on a same assumption regarding lot size: negative order quantities resulting from calculations are treated as excessive inventories which may be: (i) handed back to suppliers at no cost; or (ii) completely disregarded. Such return policy, according to the authors proposing the BE models, would not impact significantly in the magnitude of the estimated BE. In this paper we demonstrate that the return policy is key in the measurement of the BE, affecting significantly its results. We thus propose a new return policy to respond to negative order quantities with the objective of minimizing the undesirable amplification in demand variability resulting from inadequate policies adopted in response to demand levels.
\end{abstract}

\section{INTRODUÇÃO}

O primeiro trabalho conhecido sobre a amplificação da variabilidade da demanda, atribuído a Forrester (1961), apresenta uma série de estudos de caso relativos ao tema. De acordo com o autor, o atraso na transferência de informações sobre a demanda e o atraso na transferência física dos produtos ao longo de uma cadeia de abastecimento são as duas principais causas do fenômeno. Isto pode ser conseqüência da dinâmica dos processos industriais ou da mudança de comportamento das organizações que desenvolvem esses processos, tais como alterações na estrutura ou adoção de novas políticas empresariais.

De acordo com Lee et al. (1997), o termo bullwhip effect, denominação original do efeito chicote (EC), foi cunhado por executivos da Procter \& Gamble (P\&G) a partir de observações sobre as vendas das fraldas Pampers. Eles constataram que, apesar da taxa de demanda por fraldas se manter praticamente estável, ocorria um aumento na variabilidade da demanda das matérias-primas necessárias à sua produção.

\footnotetext{
1 José Carlos Fioriolli, Universidade Federal do Rio Grande do Sul. Escola de Administração. Porto Alegre, RS, Brasil (e-mail: fioriolli@producao.ufrgs.br).

${ }^{2}$ Flávio Sanson Fogliatto, Universidade Federal do Rio Grande do Sul. Programa de Pós-Graduação em Engenharia de Produção. Porto Alegre, RS, Brasil (e-mail: ffogliatto@producao.ufrgs.br).
}

Manuscrito recebido em 18/9/2007 e aprovado para publicação em 2/6/2008. Este artigo é parte de TRANSPORTES, volume XVI, número 1 , de junho de 2008. ISSN: 1415-7713.
O EC é um importante fenômeno presente em cadeias de abastecimento. Observa-se um EC quando a variabilidade da demanda aumenta na medida em que se avança nos níveis da cadeia, desde o varejista até o fabricante (Chen et al., 2003), produzindo impactos negativos sobre a regularidade e a estabilidade dos pedidos recebidos em todos os seus níveis.

A dinâmica do EC, considerando dois níveis adjacentes de uma cadeia de abastecimento, pode ser assim descrita:

(i) o pedido gerado pelo nível $k$ é definido a partir da previsão de demanda do nível anterior, $k-1$, e depende dos dados históricos relativos àquela demanda e da técnica de previsão utilizada no nível $k$;

(ii) da mesma forma, os pedidos gerados pelo nível $k+1$ são definidos em função dos dados históricos disponíveis sobre a demanda do nível anterior, $k$, e da técnica de previsão utilizada no nível $k+1$;

(iii) esta lógica se repete a cada avanço de nível, de modo que os dados disponíveis, em cada nível, com exceção do nível mais baixo da cadeia, baseiam-se em uma expectativa de demanda e não na demanda real. Esta dinâmica dificulta a realização de previsões de demanda e torna mais complexo o controle de estoques. A variabilidade da demanda no nível $k$ é maior do que no nível $k-$ 1; a variabilidade da demanda no nível $k+1$ é maior do que no nível $k$ e assim sucessivamente até o nível mais alto da cadeia. 
Vários aspectos gerenciais merecem destaque neste processo de amplificação da variabilidade da demanda. De acordo com Lee et al. (1997), Carlsson e Fullér (2000) e Chen et al. (2003), o EC potencialmente implica nas seguintes conseqüências para fabricantes, distribuidores e varejistas:

(i) conseqüências locais, tais como baixos níveis de serviço, vendas perdidas em função das rupturas de estoque geradas por variações extremas da demanda, aumentos dos estoques de segurança, aumento no número de reprogramações de produção para cobrir emergências e gestão ineficiente de recursos locais e

(ii) conseqüências sistêmicas, tais como elevação dos custos relacionados a estoques, queda do retorno sobre o capital investido, queda da produtividade dos funcionários que atuam nos processos produtivos, processo decisório reativo e gestão ineficiente dos recursos da cadeia como um todo.

Observa-se que, mesmo consideradas separadamente, as conseqüências negativas do EC constituem uma séria ameaça à qualidade da gestão empresarial.

O principal desafio no gerenciamento do EC consiste em reduzir as ineficiências acima identificadas, de modo a otimizar o uso dos recursos empregados nos diferentes níveis de uma cadeia de abastecimento. Isto depende do grau de conhecimento que se tem sobre as suas causas e sobre a sua intensidade. O estudo do EC tem representado um grande desafio para os pesquisadores em razão de seu caráter estocástico e serial. Essa complexa tarefa compreende, inicialmente, a identificação da natureza da demanda independente e a projeção dos conseqüentes níveis de demanda dependente, nos diferentes pontos da estrutura. Nesse sentido, a caracterização e a quantificação do EC tem sido um tema freqüente de pesquisa nos últimos anos. Autores como Lee et al. (1997), Cachon (1999), Carlsson e Fullér (2000), Chen et al. (2000), Fransoo e Wouters (2000) e Warburton (2004) vêm apresentando importantes desenvolvimentos teóricos sobre o tema. Um dos principais modelos de quantificação do EC, apresentado por Chen et al. (2000), é formulado como função do lead time, da variância da demanda e do número de períodos utilizados na previsão da demanda; tanto esses autores como Lee et al. (1997), ao descreverem o processo de cálculo do tamanho dos pedidos, trabalham com o pressuposto de que valores negativos correspondem a excessos de estoque que podem ser devolvidos sem custo. De modo similar, Warburton (2004) trabalha com o pressuposto de que valores negativos correspondem a excessos de estoque que podem ser considerados nulos. Estas situações podem gerar graves distorções no dimensionamento do EC, conforme desenvolvimento apresentado no presente trabalho.
Este artigo analisa o impacto produzido por diferentes políticas de tratamento dos excessos de estoque sobre a quantificação do EC em ambientes com demanda estocástica. Além da política $\mathrm{P} 1$, cujos excessos de estoque são devolvidos a custo zero, outras duas políticas são investigadas: P2 - tamanhos de pedido negativos são considerados como nulos, ou seja, não motivam nem compra nem devolução de itens, e P3 tamanhos de pedido negativos sinalizam estoques excedentes, os quais devem ser utilizados para suprir demandas futuras. Demonstra-se que a política P1, pressuposto original dos autores dos principais modelos de quantificação do EC, somente leva a uma quantificação correta do EC em cenários específicos, bastante limitados. Complementarmente, demonstra-se que a política $\mathrm{P} 2$ pode gerar aumentos desnecessários no tamanho médio dos pedidos, o que faz com que o total de unidades pedidas, ao longo do tempo, seja muito superior às reais necessidades expressas pela demanda. Por fim, sugere-se que a política P3, proposição original deste trabalho, seja capaz de contribuir para a redução da intensidade do EC, atendendo adequadamente as quantidades demandadas.

O presente artigo apresenta duas contribuições relevantes para o estudo do EC em cadeias de abastecimento. Primeiro, analisa-se detalhadamente o modelo mais referenciado de quantificação do $\mathrm{EC}$, devido a Chen et al. (2000), indicando cenários em que o mesmo mede adequadamente o fenômeno. Segundo, aprofunda-se a análise de um dos pressupostos que regem o desenvolvimento e a utilização da maioria dos modelos de quantificação do EC disponíveis na literatura (valores negativos, referentes ao tamanho dos pedidos, são tratados como excessos de estoque que podem ser devolvidos sem custo ou considerados nulos). Os desenvolvimentos no artigo demonstram que, além de resultar em uma quantificação imprecisa do EC, tal pressuposto não representa a realidade da prática da gestão de cadeias de abastecimento. Para contornar essa deficiência, o artigo finaliza por propor uma política mais realista para o tratamento destes excessos.

O restante do artigo está organizado em sete seções. $\mathrm{Na}$ seção 2 são apresentadas as principais definições do EC. A seção 3 contém o detalhamento dos principais modelos de quantificação do EC disponíveis na literatura. Os mesmos modelos são analisados criticamente na seção 4 . Na seção 5 apresentam-se políticas de tratamento de excessos de estoque em cadeias de suprimento e seus impactos sobre a quantificação do EC. Especial destaque é dado à política P3 (tamanhos de pedido negativos sinalizam estoques excedentes, os quais podem ser utilizados para suprir demandas futuras). Na seção 6, analisam-se os efeitos da adoção de diferentes políticas de tratamento de excessos de estoque sobre a quantificação do EC, utilizando o modelo 
de Chen et al. (2000) como base para a análise comparativa. As conclusões do artigo são apresentadas na seção 7 .

\section{EFEITO CHICOTE}

O Quadro 1 apresenta as principais definições do EC, agrupadas de acordo com o tipo de relação observada entre as variáveis demanda e pedido.

Diversos autores têm desenvolvido pesquisas para comprovar a existência do EC e para identificar suas causas e reduzir seus efeitos sobre estoques, custos e níveis de serviço. Segundo Lee e Billington (1992), a complexidade das cadeias de abastecimento pode se constituir em uma grande oportunidade para se obter ganhos em eficiência operacional. Os autores analisam quatorze possíveis falhas, e algumas oportunidades delas decorrentes, na gestão de estoques. Três delas se relacionam diretamente com a amplificação da demanda: a ineficiência dos sistemas de informação, a imprecisão dos dados sobre entregas e a desconsideração dos impactos gerados pelas incertezas na demanda e no lead time de entrega de pedidos. Lee et al. (1997) identificaram e descreveram quatro causas do EC, destacando o fato de que as informações transferidas ao longo da cadeia de abastecimento sob a forma de pedidos tendem a ser distorcidas e podem desorientar os tomadores de decisão em cada um dos níveis da estrutura. Baganha e Cohen (1998) desenvolveram um modelo para analisar o efeito estabilizador dos estoques frente às variações da demanda. Esses autores concluíram que, embora investimentos em estoques sejam justificados por seu papel estabilizador, nem sempre isto ocorre na prática. Dependendo das políticas de controle de estoques adotadas pode haver uma amplificação das variações ao invés de uma redução.

Vários modelos foram desenvolvidos para estudar o comportamento dos estoques em situações em que a informação sobre a demanda não é conhecida por todos os membros da cadeia. Chen et al. (2000) propõem um modelo de cadeia de abastecimento com dois estágios em que a demanda é modelada como um processo auto-regressivo de primeira ordem; nesse trabalho, os autores assumem que no início de cada período de pedido o varejista usa a técnica da média móvel simples para gerar estimativas sobre a média e o desvio-padrão da demanda, que servem de base para

Quadro 1: Principais definições do EC

\begin{tabular}{|c|c|c|}
\hline Tipo & Autor(es) & Definição do EC \\
\hline \multirow{4}{*}{$\begin{array}{l}\text { Relação entre a variância dos pedidos e a } \\
\text { variância da demanda }\end{array}$} & $\begin{array}{l}\text { Lee, Padmanabhan e Whang } \\
\text { (1997) }\end{array}$ & $\begin{array}{l}\text { Fenômeno presente em estruturas de abastecimento em que os } \\
\text { pedidos enviados aos fornecedores tendem a apresentar variância } \\
\text { maior que a variância das vendas realizadas junto aos clientes } \\
\text { (distorção da demanda); esta distorção se propaga para os outros } \\
\text { níveis da estrutura de forma amplificada (amplificação da variân- } \\
\text { cia). }\end{array}$ \\
\hline & Cachon (1999) & $\begin{array}{l}\text { Propagação da variância da demanda ao longo de uma cadeia de } \\
\text { abastecimento. }\end{array}$ \\
\hline & Carlsson e Fullér (2000) & $\begin{array}{l}\text { Fenômeno em que o tamanho dos pedidos encaminhados aos } \\
\text { fornecedores tende a ter maior variância que as quantidades ven- } \\
\text { didas aos clientes (distorção da demanda) e, na seqüência, a vari- } \\
\text { ância do tamanho dos pedidos encaminhados aos fabricantes é } \\
\text { ainda maior que a variância das quantidades vendidas aos clientes. } \\
\text { Propagação da distorção da demanda de uma forma amplificada } \\
\text { (amplificação da variância). }\end{array}$ \\
\hline & $\begin{array}{l}\text { Chen, Drezner, Ryan e } \\
\text { Simchi-Levi (2000) }\end{array}$ & $\begin{array}{l}\text { Aumento da variabilidade da demanda ao longo de uma cadeia de } \\
\text { abastecimento. }\end{array}$ \\
\hline $\begin{array}{l}\text { Relação entre a taxa de pedidos e a taxa da } \\
\text { demanda }\end{array}$ & Warburton (2004) & $\begin{array}{l}\text { Amplificação da variabilidade da taxa de pedidos ao longo de uma } \\
\text { cadeia de abastecimento. }\end{array}$ \\
\hline $\begin{array}{l}\text { Relação entre o coeficiente de variação da } \\
\text { demanda gerada por um nível da cadeia de } \\
\text { abastecimento e o coeficiente de variação } \\
\text { da demanda recebida por este nível }\end{array}$ & Fransoo e Wouters (2000) & $\begin{array}{l}\text { Aumento da razão entre o coeficiente de variação dos pedidos e o } \\
\text { coeficiente de variação da demanda ao longo da cadeia de abaste- } \\
\text { cimento. }\end{array}$ \\
\hline
\end{tabular}


determinar o tamanho do pedido. Chen et al. (2000) investigam em que grau a variabilidade dos pedidos é maior que a variabilidade da demanda real. Watson e Zheng (2001) analisam uma cadeia de abastecimento utilizando um modelo de demanda correlacionada para mostrar que os gerentes tendem a reagir desproporcionalmente, o que acentua as variações da demanda, e concluem que isto leva à prática de políticas subótimas de reabastecimento de estoques, causando o EC. Um estudo experimental baseado no Beer Distribution Game (BDG) foi conduzido por Croson e Donohue (2002) para identificar os fatores comportamentais que influenciam o EC; esses mesmos autores realizaram um experimento para estudar o impacto do compartilhamento de informações no POS (Point of Sale ou Ponto de Venda) como fator redutor do EC (CROSON; DONOHUE, 2003).

\section{PRINCIPAIS MODELOS DE QUANTIFICAÇÃO DO EFEITO CHICOTE}

Apesar da existência de vários trabalhos sobre o EC, são poucos os pesquisadores que abordam a sua quantificação. Os principais estudos realizados com vistas à mensuração do EC, revisados neste artigo, devem-se a Lee et al. (1997), Chen et al. (2000), Fransoo e Wouters (2000) e Warburton (2004). Chen e Disney (2003) e Hosoda e Disney (2004) propuseram abordagens alternativas para a quantificação do EC, operacionalizadas através de pequenas modificações nos modelos existentes; tais abordagens não são detalhadas nesta seção. Outros autores desenvolveram trabalhos complementares sobre o tema, sendo os principais relatados na seqüência.

Anderson et al. (2000) criaram o Mortgage Service Game (MSG) com o objetivo de simular os processos decisórios próprios de uma cadeia de abastecimento. Nesse modelo, estudantes decidem sobre produção e estoques com vistas à redução do EC. Cachon (1999) indica que é possível reduzir o EC através de uma programação de pedidos baseada em lotes menores. Kunková (2002) mostra que a quantificação do EC depende da forma de agregação dos dados sobre a demanda e conclui que o EC pode se manifestar de duas formas: diretamente, através da amplificação da demanda e, indiretamente, através da elevação dos níveis de estoques. Sancar (2003), ao realizar uma revisão sobre os modelos de quantificação do EC, mostra que a amplificação da variabilidade da demanda pode ser reduzida através de medidas gerenciais (ex.: coordenação e estabilização de preços e compartilhamento de informações), mas não pode ser totalmente eliminada.

Alguns autores, como Moyaux (2000), Derrick
(2003) e Donovan (2003) utilizam simulações para avaliar o EC e estabelecer procedimentos voltados à redução desse fenômeno. Moyaux, Chaib-draa e D'amours (2003) elaboraram um jogo denominado Québec Wood Supply Game (QWSG), adaptado do $\mathrm{BDG}$, para simular o EC no setor florestal. A simulação foi programada em planilha de cálculo, utilizando multi-agentes para desenvolver os processos decisórios de quantificação e processamento de pedidos, produção, transporte e armazenamento de produtos. Multi-agentes são estruturas que reproduzem o comportamento dos indivíduos com base em um conjunto de regras; o efeito combinado das decisões tomadas por multi-agentes serve para simular os processos decisórios empresariais, inclusive em relação a estruturas hierárquicas, a exemplo das cadeias de abastecimento. O objetivo principal dos pesquisadores consiste em aumentar a eficiência de uma cadeia de abastecimento através da redução da variabilidade da demanda, mantendo os estoques em seus níveis mais baixos, oferecendo altos níveis de serviço aos consumidores e incorporando ao modelo as causas do EC apontadas por Lee et al. (1997). A simulação evidenciou a complexidade das decisões orientadas para a eficiência em ambientes sujeitos ao EC.

De acordo com o tipo de relação observada entre as variáveis demanda e pedido (Quadro 1), as principais abordagens utilizadas para a quantificação do EC podem ser divididas em três grupos:

(i) cálculo da relação entre a variância dos pedidos e a variância da demanda (Lee et al., 1997; Chen et al., 2000);

(ii) cálculo do quociente entre o coeficiente de variação da demanda gerada por um nível da cadeia e o coeficiente de variação da demanda recebida por este mesmo nível (Fransoo e Wouters, 2000) e

(iii) cálculo da relação entre a taxa de pedidos e a taxa da demanda (Warburton, 2004).

Os modelos referidos acima são detalhados na seqüência (a notação utilizada é apresentada no Quadro 2).

\subsection{Modelo de Lee, Padmanabhan e Whang (1997)}

O processo de formação da demanda é definido utilizando um modelo auto-regressivo de primeira ordem, com a seguinte forma:

$$
D_{t}=d+\rho D_{t-1}+\varepsilon_{t}
$$

Calcula-se o tamanho do pedido somando a diferença entre os estoques-alvo dos períodos $t$ e $t-1$ com a demanda do período $t-1$

$$
Q_{t}=A_{t}-A_{t-1}+D_{t-1}
$$


O desenvolvimento algébrico realizado pelos autores do modelo permite definir o tamanho do pedido a ser colocado em função do parâmetro de correlação da demanda e do lead time, da seguinte maneira:

$$
\begin{aligned}
& Q_{t}=A_{t}-A_{t-1}+D_{t-1} \\
& Q_{t}=\frac{\rho\left(1-\rho^{L+1}\right)}{1-\rho}\left(D_{t-1}-D_{t-2}\right)+D_{t-1}
\end{aligned}
$$

A variância dos pedidos é resultante da soma de duas parcelas: a primeira diretamente dependente da variância da demanda e a outra conjuntamente dependente da estrutura da demanda e da magnitude do lead time.

$$
\begin{aligned}
\operatorname{Var}\left(Q_{t}\right)= & \operatorname{Var}\left(D_{t-1}\right)+\frac{2 \rho\left(1-\rho^{L+1}\right)\left(1-\rho^{L+2}\right)}{(1+\rho)(1-\rho)^{2}} \\
& >\operatorname{Var}\left(D_{t-1}\right)
\end{aligned}
$$

O EC é calculado através da relação entre a variância dos pedidos e a variância da demanda. A forma final do modelo proposto por Lee et al. (1997) é a seguinte:

$$
\begin{aligned}
& E C_{t, t-1}=\frac{\operatorname{Var}\left(Q_{t}\right)}{\operatorname{Var}\left(D_{t-1}\right)} \\
& E C_{t, t-1}=1+\frac{2 \rho\left(1-\rho^{L+1}\right)\left(1-\rho^{L+2}\right)}{(1+\rho)(1-\rho)^{2}}\left(\frac{1}{\operatorname{Var}\left(D_{t-1}\right)}\right)
\end{aligned}
$$

\subsection{Modelo de Chen, Drezner, Ryan e Simchi- Levi (2000)}

Chen et al. (2000) consideram uma cadeia de abastecimento em que a cada período $t$ um varejista observa seu nível de estoque e coloca um pedido $Q_{t}$ para um fabricante. Após o pedido ter sido colocado, a demanda $D_{t}$ relativa ao período em curso é atendida caso haja estoque suficiente; demandas não atendidas ficam pendentes.

Um lead time $L$, constante, transcorre entre o momento em que o pedido é colocado pelo varejista e o momento em que o pedido é recebido por ele, de modo que um pedido colocado no fim de um período $t$ é recebido no início do período $t+L$.

A demanda visualizada pelo varejista é uma variável aleatória, cujo processo de formação é representa-

\begin{tabular}{|c|c|c|c|}
\hline Símbolo & Descrição & Símbolo & Descrição \\
\hline$t$ & $\begin{array}{l}\text { Subscrito que denota o momento de observação } \\
\text { da variável no tempo }\end{array}$ & $Q_{t}$ & Tamanho do pedido em $t$ \\
\hline$L$ & Lead time & $k$ & Nível hierárquico da estrutura em análise \\
\hline$p$ & $\begin{array}{l}\text { Número de períodos utilizados no cálculo da } \\
\text { média móvel da demanda }\end{array}$ & $\theta_{D, L}^{i n}$ & $\begin{array}{l}\text { Coeficiente de variação da demanda recebi- } \\
\text { da durante o lead time }\end{array}$ \\
\hline$d$ & $\begin{array}{l}\text { Constante não negativa, utilizada no modelo } \\
\text { auto-regressivo do processo de formação da } \\
\text { demanda }\end{array}$ & $\theta_{D, L}^{\text {out }}$ & $\begin{array}{l}\text { Coeficiente de variação da demanda gerada } \\
\text { durante o lead time }\end{array}$ \\
\hline$\rho$ & $\begin{array}{l}\text { Parâmetro de correlação do processo de forma- } \\
\text { ção da demanda }\end{array}$ & $I(t)$ & Nível do estoque em $t$ \\
\hline$\varepsilon_{t}$ & $\begin{array}{l}\text { Erro aleatório em } t \text {, normalmente distribuído, } \\
\text { com média } 0 \text { e desvio-padrão } \sigma\end{array}$ & $I_{0}$ & Estoque inicial \\
\hline$D_{t}$ & Demanda estocástica em $t$ & $R(t)$ & Taxa de recebimento em $t$ \\
\hline$D_{i n}(t, t+L)$ & Demanda recebida durante o lead time & $D(t)$ & Taxa de demanda em $t$ \\
\hline$\mu\left[D_{i n}(t, t+L)\right]$ & Média da demanda recebida durante o lead time & $T$ & Ajuste temporal \\
\hline$\sigma\left[D_{i n}(t, t+L)\right]$ & $\begin{array}{l}\text { Desvio-padrão da demanda recebida durante o } \\
\text { lead time }\end{array}$ & $O(t)$ & Taxa de pedido em $t$ \\
\hline$D_{\text {out }}(t, t+L)$ & Demanda gerada durante o lead time & $W$ & Função $W$ de Lambert \\
\hline$\mu\left[D_{\text {out }}(t, t+L)\right]$ & Média da demanda gerada durante o lead time & $B$ & Coeficiente da função $W$ de Lambert \\
\hline$\sigma\left[D_{\text {out }}(t, t+L)\right]$ & $\begin{array}{l}\text { Desvio-padrão da demanda gerada durante o } \\
\text { lead time }\end{array}$ & $a$ & Parâmetro da parte real de $B$ \\
\hline$\hat{D}_{L, t}$ & $\begin{array}{l}\text { Estimativa da demanda média durante o lead } \\
\text { time, em } t\end{array}$ & $\alpha$ & Parâmetro da parte complexa de $B$ \\
\hline$e_{t}$ & Erro de previsão da demanda em $t$ & $J$ & $\begin{array}{l}\text { Fator de ponderação utilizado no cálculo de } \\
a\end{array}$ \\
\hline$C_{L, p}$ & Função constante de $L, \rho$ e $p$ & $K$ & $\begin{array}{l}\text { Fator de ponderação utilizado no cálculo de } \\
\alpha\end{array}$ \\
\hline$\hat{\sigma}_{e, t}^{L}$ & $\begin{array}{l}\text { Estimativa do desvio-padrão do erro de previsão } \\
\text { de demanda durante o lead time, em } t\end{array}$ & $\omega$ & $\begin{array}{l}\text { Parâmetro da parte real da função } W \text { de } \\
\text { Lambert }\end{array}$ \\
\hline$z$ & $\begin{array}{l}\text { Constante correspondente ao nível de serviço } \\
\text { desejado }\end{array}$ & $\Omega$ & $\begin{array}{l}\text { Parâmetro da parte complexa da função } W \text { de } \\
\text { Lambert }\end{array}$ \\
\hline$A_{t}$ & Estoque-alvo (order-up-to) em $t$ & & \\
\hline
\end{tabular}
do por um modelo auto-regressivo de primeira ordem:

$$
D_{t}=d+\rho D_{t-1}+\varepsilon_{t}
$$

O varejista segue uma política order-up-to em que o

Quadro 2: Notação utilizada nos modelos de quantificação do EC 
estoque-alvo é calculado a partir da estimativa da demanda durante o lead time, da constante $\mathrm{z}$ associada ao nível de serviço desejado e da estimativa do desvio-padrão do erro de previsão da demanda durante o lead time:

$$
A_{t}=\hat{D}_{L, t}+z \hat{\sigma}_{e, t}^{L}
$$

onde $\hat{D}_{L, t}=L\left(\sum_{i=1}^{p} D_{t-1} / p\right) \mathrm{e}$

$$
\hat{\sigma}_{e, t}^{L}=C_{L, p}\left(\sum_{i=1}^{p}\left(e_{t-i}\right)^{2} / p\right)^{1 / 2} \text {. }
$$

O tamanho do pedido é calculado em função do estoque-alvo e da demanda; eventuais valores negativos são assumidos como excessos de estoque que podem ser devolvidos sem custo, conforme proposto por Kahn (1987) e Lee et al. (1997).

$$
Q_{t}=A_{t}-A_{t-1}+D_{t-1}
$$

O cálculo do pedido pode ser apresentado em função das estimativas da demanda durante o lead time, da constante vinculada ao nível de serviço desejado, das estimativas dos desvios dos erros de previsão relativos ao mesmo período e da demanda observada no período anterior.

$$
\begin{gathered}
Q_{t}=\hat{D}_{L, t}-\hat{D}_{L, t-1}+z\left(\hat{\sigma}_{e, t}^{L}-\hat{\sigma}_{e, t-1}^{L}\right)+D_{t-1} \\
Q_{t}=L\left(\frac{D_{t-1}-D_{t-p-1}}{p}\right)+D_{t-1}+z\left(\hat{\sigma}_{e, t}^{L}-\hat{\sigma}_{e, t-1}^{L}\right) \\
Q_{t}=(1+L / p) D_{t-1}-(L / p) D_{t-p-1}+z\left(\hat{\sigma}_{e, t}^{L}-\hat{\sigma}_{e, t-1}^{L}\right)
\end{gathered}
$$

Pela definição do EC, segue que:

$$
E C=\frac{\operatorname{Var}(Q)}{\operatorname{Var}(D)} \geq 1+\left(\frac{2 L}{p}+\frac{2 L^{2}}{p^{2}}\right)\left(1-\rho^{p}\right)
$$

No caso específico de não existir correlação entre as demandas período a período, ou seja, quando $\rho=0$ :

$$
E C=\frac{\operatorname{Var}(Q)}{\operatorname{Var}(D)} \geq 1+\frac{2 L}{p}+\frac{2 L^{2}}{p^{2}}
$$

\subsection{Modelo de Fransoo e Wouters (2000)}

Fransoo e Wouters (2000) quantificam a amplificação da variabilidade da demanda como sendo o quociente entre o coeficiente de variação da demanda gerada em um nível da estrutura e o coeficiente de variação da demanda recebida por este nível, ambos definidos no período correspondente ao lead time.

$$
\begin{gathered}
E C=\theta_{D, L}^{\text {out }} / \theta_{D, L}^{\text {in }} \\
\theta_{D, L}^{\text {out }}=\frac{\sigma\left[D_{\text {out }}(t, t+L)\right]}{\mu\left[D_{\text {out }}(t, t+L)\right]}
\end{gathered}
$$

$$
\begin{gathered}
\theta_{D, L}^{\text {in }}=\frac{\sigma\left[D_{\text {in }}(t, t+L)\right]}{\mu\left[D_{\text {in }}(t, t+L)\right]} \\
E C=\frac{\sigma\left[D_{\text {out }}(t, t+L)\right] \mu\left[D_{\text {in }}(t, t+L)\right]}{\sigma\left[D_{\text {in }}(t, t+L)\right] \mu\left[D_{\text {out }}(t, t+L)\right]}
\end{gathered}
$$

A expressão pode ser generalizada para uma cadeia de abastecimento com $k$ níveis, da seguinte forma:

$$
\begin{aligned}
E C_{1, k} & =\left(\theta_{D, L, 1}^{\text {out }} \theta_{D, L, 2}^{\text {out }} \ldots \theta_{D, L, k}^{\text {out }}\right) /\left(\theta_{D, L, 1}^{\text {in }} \theta_{D, L, 2}^{\text {in }} \ldots \theta_{D, L, k}^{\text {in }}\right) \\
E C_{1, k} & =\theta_{D, L, k}^{\text {out }} / \theta_{D, L, 1}^{\text {in }}
\end{aligned}
$$

\subsection{Modelo de Warburton (2004)}

$\mathrm{Na}$ construção do modelo, Warburton (2004) utiliza um processo de formação de demanda definido por um modelo auto-regressivo de primeira ordem, similar àqueles utilizados por Lee et al. (1997) e Chen et al. (2000):

$$
D_{t}=d+\rho D_{t-1}+\varepsilon_{t}
$$

O estoque é esvaziado pela taxa de demanda e aumentado pela taxa de recebimento; deste modo, a equação de balanço do estoque é:

$$
d I / d t=R(t)-D(t)
$$

Utilizando uma política onde os pedidos são proporcionais aos déficits dos estoques e incorporando uma variável de ajuste temporal $T$ (para ajustar a taxa de pedido, com base na premissa de que a recuperação do déficit do estoque pode ser feita, continuamente, ao longo do tempo), tem-se:

$$
\begin{aligned}
& O(t)=[A-I(t)] / T \text { se } I(t)<A \text { e } \\
& O(t)=0, \text { em caso contrário }
\end{aligned}
$$

Os itens recebidos apresentam uma defasagem temporal $L$ constante em relação aos itens demandados (lead time),

$$
R(t)=O(t-L)
$$

Substituindo a política de pedidos na Equação (20), tem-se:

$$
\frac{d I}{d t}+\frac{I(t-L)}{T}=\frac{A}{T}-d
$$

Durante o lead time nenhum item é recebido, então a Equação (23) pode ser simplificada como segue:

$$
\begin{aligned}
& \text { para } t \leq L \Rightarrow R(t)=0 \\
& \log 0 d I / d T=-d \Rightarrow I(t)=I_{o}-d t
\end{aligned}
$$

Considerando que o estoque é menor que o valor estabelecido para o estoque-alvo: 


$$
O(t)=\frac{A-\left(I_{0}-d t\right)}{T}=\frac{A-I_{0}}{T}+\frac{t d}{T}
$$

A solução da Equação (23) contém a função $W$ de Lambert e corresponde a:

$$
\begin{gathered}
I(t)=I_{0}-d t \quad \text { quando } t \leq L \\
I(t)=A-d T+B \exp (W t / L) \quad \text { quando } t>L \\
\operatorname{com} B=a+i \alpha \text { e } \\
W=W(-L / T)=\omega+i \Omega \\
\alpha=\left[e^{-\omega} / \Omega\right][J(\Omega \cos \Omega+\omega \operatorname{sen} \Omega)-K \operatorname{sen} \Omega] \\
\alpha=\left[e^{-\omega} / \Omega\right][J(\omega \cos \Omega+\Omega \operatorname{sen} \Omega)-K \cos \Omega] \\
\text { sendo } J=I_{0}-A+d(T-L) \text { e } \\
K=L\left[\left(A-I_{0}\right) / T-d\right]
\end{gathered}
$$

O EC é determinado pela razão entre as taxas relativas aos pedidos que são enviados por um determinado nível da estrutura e os pedidos que chegam a este nível. Estas taxas atingem os seus valores máximos logo após $L$ períodos; deste modo, este é o momento adequado para estabelecer a razão entre elas, como segue:

$$
E C=\frac{O(L)}{d}=\frac{A-I(L)}{T d}=\frac{L d}{T d}=\frac{L}{T}
$$

$\mathrm{O}$ ajuste temporal $(T)$ que faz o estoque retornar ao seu valor desejado (estoque-alvo) pode ser obtido calculando-se o quociente entre o dobro da defasagem temporal $L$ (lead time) e $\pi(3,1415 \ldots)$. Isto significa que um EC em torno de 1,5 é praticamente inevitável, sob pena de se incorrer em freqüentes rupturas de estoque.

\section{ANÁLISE CRÍTICA DOS MODELOS APRESENTADOS}

Como pode ser constatado a partir da Equação (1), o modelo de Lee et al. (1997) tem como principal característica a simplicidade. Construído a partir de um modelo auto-regressivo de formação da demanda, incorpora o fator de correlação da demanda no tempo, o lead time e a variabilidade da demanda de entrada. $\mathrm{Na}$ prática, a identificação desse fator de correlação tende a ser imprecisa, o que enfraquece o modelo. Apresenta, ainda, um erro de formulação referente ao fator de correlação, pois se este for negativo (caso de correlação negativa, próxima a-1) o EC pode resultar negativo. Dado que o EC é a razão entre duas variâncias, este resultado seria incorreto, por definição.

O modelo de Chen et al. (2000) é um dos mais cita- dos na literatura. Foi construído a partir de um modelo auto-regressivo que representa o processo de formação da demanda. De acordo com a Equação (13), torna-se evidente a importância da escolha de um número adequado de períodos para o cálculo da média móvel a ser utilizada nas estimativas da demanda, com vistas à redução do EC. Ao tratar os valores negativos obtidos no cálculo dos pedidos como excessos de estoque que podem ser devolvidos sem custo, o modelo tende a superdimensionar o EC.

O modelo de Fransoo e Wouters (2000), apresentado na Equação (18), foi construído diretamente sobre a definição do EC. Seu ponto positivo é a incorporação dos coeficientes de variação da demanda de entrada e da demanda de saída, para cada nível da estrutura. $\mathrm{O}$ aspecto negativo reside no fato de que o modelo não mostra como são calculados o desvio-padrão e a média da demanda de saída.

A formulação final do modelo de Warburton (2004), conforme apresentado na Equação (28), é simples, porém de desenvolvimento relativamente complexo. $\mathrm{O}$ aspecto positivo consiste na incorporação, ao lead time, de uma variável de ajuste temporal para controlar a taxa de pedido, o que é compatível com a natureza estocástica do EC. Sua fragilidade reside na linearização do fenômeno em relação ao lead time.

\section{POLÍTICAS DE TRATAMENTO DE EXCESSOS DE ESTOQUE E SEUS IMPACTOS SOBRE O EC}

O valor correspondente ao tamanho do pedido, calculado em função do estoque-alvo e da demanda [de acordo com as Equações (2) e (8)] pode ser negativo; neste caso, o resultado é assumido como excesso de estoque que pode ser devolvido sem custos (política P1), segundo Kahn (1987), Lee et al. (1997) e Chen et al. (2000). Quando calculado em função do estoquealvo, do nível de estoque e do ajuste temporal [de acordo com as Equações (21a) e (21b)], eventuais valores negativos são considerados nulos (política P2); neste caso, não motivam nem compra nem devolução de itens. Em ambientes com lead time constante, de acordo com Lee et al. (1997), Chen et al. (2000) e Warburton (2004), esses valores negativos não causam impactos significativos na intensidade do EC. Entretanto, os resultados obtidos nesta pesquisa indicam que a ocorrência de valores negativos (relativos ao tamanho dos pedidos) tende a produzir graves distorções no dimensionamento do EC, mesmo em ambientes com lead time constante.

Através de um exemplo numérico (Tabela 1) são apresentadas três políticas de tratamento dos excessos de estoque, com vistas à identificação dos respectivos impactos sobre o EC: 
(i) devolução dos excessos de estoque sem custo [política P1, adotada por Lee et al. (1997) e Chen et al. (2000)];

(ii) desconsideração dos excessos de estoque [política P2, adotada por Warburton (2004)]; e

(iii) aproveitamento dos excessos de estoque no cálculo do tamanho dos pedidos dos períodos seguintes (política $\mathrm{P} 3$, proposta nesta pesquisa).

Dependendo da política escolhida, o pedido ajustado poderá assumir valores diferentes do pedido original. Ao adotar a política P1, o tamanho do pedido permanece inalterado, independentemente de seu valor ser positivo ou negativo. Desta forma, o pedido ajustado $Q_{t}^{R}$ é igual ao pedido original:

$$
Q_{t}^{R}=Q_{t}
$$

Caso a política adotada seja $\mathrm{P} 2$, o tamanho do pedido será ajustado (para zero) somente se apresentar valores negativos:

$$
Q_{t}^{R}=\max \left\{0 ; Q_{t}\right\}
$$

Na política P3, o ajuste no tamanho do pedido dependerá do estoque em excesso, $H_{t}$, como segue.

$$
\begin{gathered}
H_{t}=\operatorname{abs}\left[\min \left\{0 ; Q_{t}-H_{t-1}\right\}\right] \\
Q_{t}^{R}=\max \left\{0 ; Q_{t}-H_{t-1}\right\}
\end{gathered}
$$

Diferentes tratamentos dos excessos de estoque produzem diferentes resultados no cálculo do tamanho dos pedidos, conforme a Tabela 1. Dada a possibilidade de ocorrência de valores negativos para o tamanho dos pedidos, a política P1 deixa de ser realista. Nestas condições, o desvio-padrão dos pedidos tende a aumentar expressivamente, o que distorce a quantificação do EC. No caso da política P2, ocorre um aumento expressivo do tamanho médio dos pedidos, pois os valores negativos são totalmente desprezados; esta situação também é pouco realista, uma vez que o total de pedidos, ao longo do tempo, é muito superior às reais necessidades expressas pela demanda (excesso de $25 \%$ no tamanho médio dos pedidos, no exemplo apresentado).

A política $\mathrm{P} 3$, além de manter a média dos pedidos em níveis adequados à demanda e aos estoques-alvo desejados, não inflaciona o desvio-padrão dos pedidos. Todos os excessos de estoque, na política P3, são considerados no cálculo do tamanho dos pedidos seguintes. Com a adoção da política $\mathrm{P} 3$, o ajuste realizado nos pedidos em função dos excessos de estoque reduz o desvio-padrão da série original de pedidos, sem alterar a sua média, conforme exemplo apresentado na Tabela 1.

\section{SIMULAÇÕES DO EC versus MODELO DE CHEN et al. (2000)}

As simulações do EC, relativas às políticas $\mathrm{P} 1, \mathrm{P} 2$ e P3, foram realizadas utilizando a planilha de cálculo EFEITO_CHICOTE.xls (gerada em Excel ${ }^{\circledR}$, com add-in da Crystal Ball ${ }^{\circledR}$, disponível no endereço http://portals.crystalball.com/Downloads) criada especialmente para esta pesquisa e disponível no endereço http://www.savefile.com/files/1396388. Inicialmente, trabalhou-se na identificação das variáveis (média e desvio-padrão da demanda, lead time e número de períodos) e dos parâmetros a serem utilizados nas simulações. Diversos cenários foram considerados, de acordo com as combinações obtidas a partir dos valores da Tabela 2.

Para cada um dos 880 cenários, foram realizadas 2000 rodadas da rotina de simulação do EC (quantificação do fenômeno e cálculo do tamanho médio dos pedidos, relativos às três políticas consideradas: P1, P2 e P3). Utilizou-se, em cada rodada, uma série de 1200 valores de demanda, gerados aleatoriamente. Os resultados obtidos indicam que há diferenças importantes no desempenho das políticas analisadas, quando comparados com os valores obtidos através do modelo de Chen et al. (2000). Esse modelo, por utilizar a política P1 de tratamento dos excessos de estoque, não adota mecanismos de ajuste no tamanho dos pedidos quando estes apresentam valores negativos, o que o

Tabela 1: Políticas de tratamento dos excessos de estoque

\begin{tabular}{cccccccccc}
\hline$t$ & $A_{t}$ & $A_{t-1}$ & $D_{t-1}$ & $Q_{t}$ & $H_{t-1}$ & $H_{t}$ & $Q_{t}^{R}$ & $Q_{t}^{R}$ & $Q_{t}^{R}$ \\
& & & & & & & P1 & P2 & P3 \\
\hline 1 & 160 & - & - & 160 & - & 0 & 160 & 160 & 160 \\
2 & 140 & 160 & 15 & -5 & 0 & 5 & -5 & 0 & 0 \\
3 & 200 & 140 & 45 & 105 & 5 & 0 & 105 & 105 & 100 \\
4 & 80 & 200 & 50 & -70 & 0 & 70 & -70 & 0 & 0 \\
5 & 100 & 80 & 30 & 50 & 70 & 20 & 50 & 50 & 0 \\
6 & 110 & 100 & 50 & 60 & 20 & 0 & 60 & 60 & 40 \\
Média & 131,6 & - & 38 & 50 & - & 15,8 & 50 & 62,5 & 50 \\
Desvio-padrão & 44,0 & - & 15,2 & 80,8 & - & 27,6 & 80,8 & 62,1 & 66,6 \\
\hline
\end{tabular}


habilita a ser usado como referência nesta análise comparativa. Acredita-se que resultados similares seriam obtidos utilizando o modelo de Lee et al. (1997), também baseado na política P1.

Tabela 2: Variáveis e valores utilizados nas simulações

\begin{tabular}{cccc}
\hline$\mu_{D}$ & $\sigma_{D}$ & $L$ & $p$ \\
\hline 1000 & 100 & 2 & 1 \\
2000 & 300 & 4 & 2 \\
3000 & 600 & 7 & 4 \\
4000 & 1000 & 10 & 6 \\
& & 14 & 8 \\
& & & 10 \\
& & & 12 \\
& & & 16 \\
& & & 16 \\
\hline
\end{tabular}

O Gráfico 1 apresenta os valores do EC obtidos através do modelo de Chen et al. (2000) e os valores obtidos por simulação para a política $\mathrm{P} 3$, considerando a totalidade dos cenários. Observa-se que em parte destes cenários há diferenças expressivas entre esses valores. Essas diferenças, geradas primariamente em função da ocorrência de valores negativos para o tamanho dos pedidos, tendem a aumentar na medida em que a relação entre o lead time médio e o número de períodos utilizados para calcular a média móvel da demanda aumenta.

O modelo de Chen et al. (2000) comporta-se bem em situações onde o quociente entre o lead time e o número de períodos é pequeno, ou seja, quando o primeiro valor é menor ou igual ao segundo valor. Quando o lead time médio é grande e o número de períodos é pequeno, a incidência de pedidos com tamanho negativo (assumidos como excessos de estoque que podem ser devolvidos a custo zero) aumenta consideravelmente e amplifica desnecessariamente a variabilidade dos pedidos, o que leva a um superdimensionamento do EC. Neste caso, os valores podem ser 20 vezes maiores em relação aos valores simulados, conforme o Gráfico 2.

A análise dos dados apresentados no Gráfico 2 indica que o EC sofre uma forte amplificação nas situações em que os pedidos não são ajustados para contemplar uma política adequada de tratamento dos excessos de estoque.

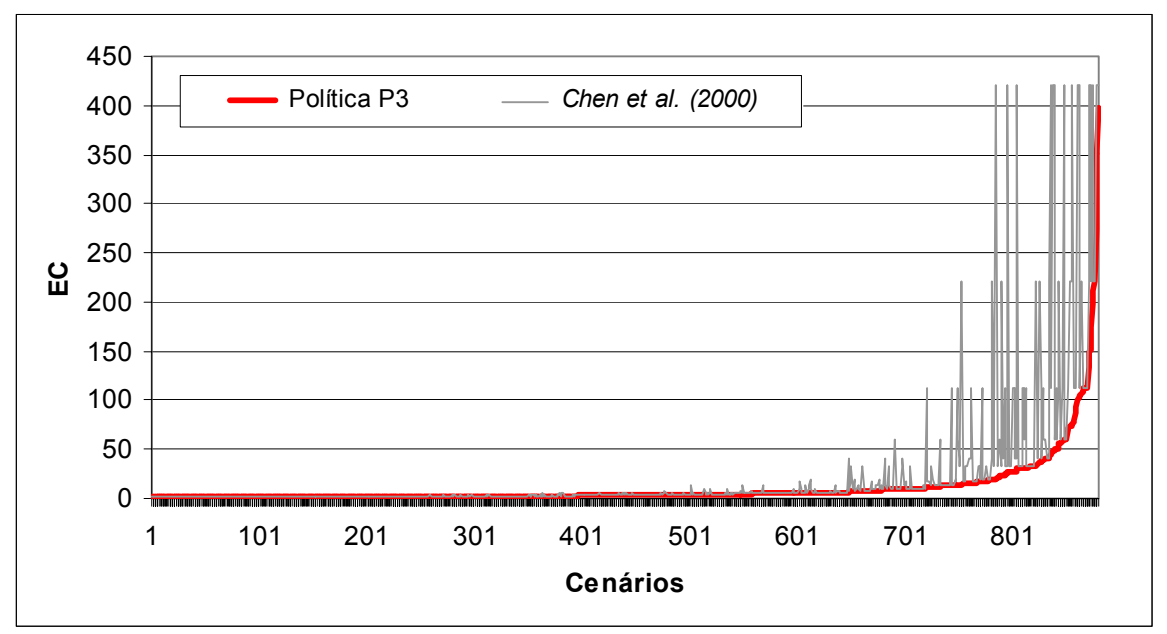

Gráfico 1: EC dimensionado para cenários com lead time constante

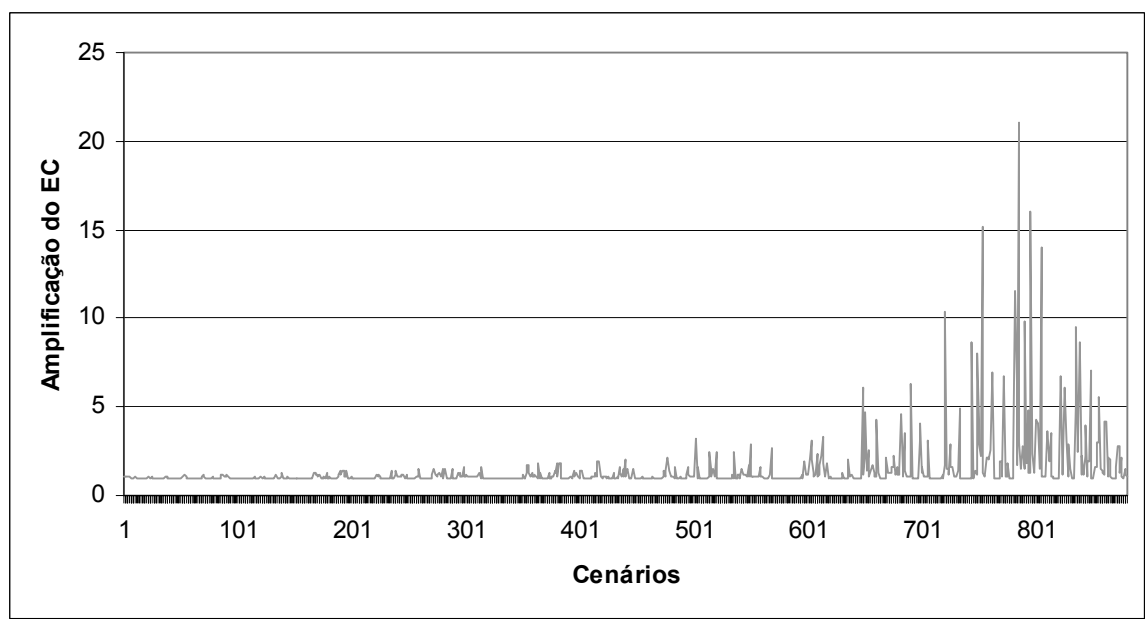

Gráfico 2: Fator de amplificação do EC 


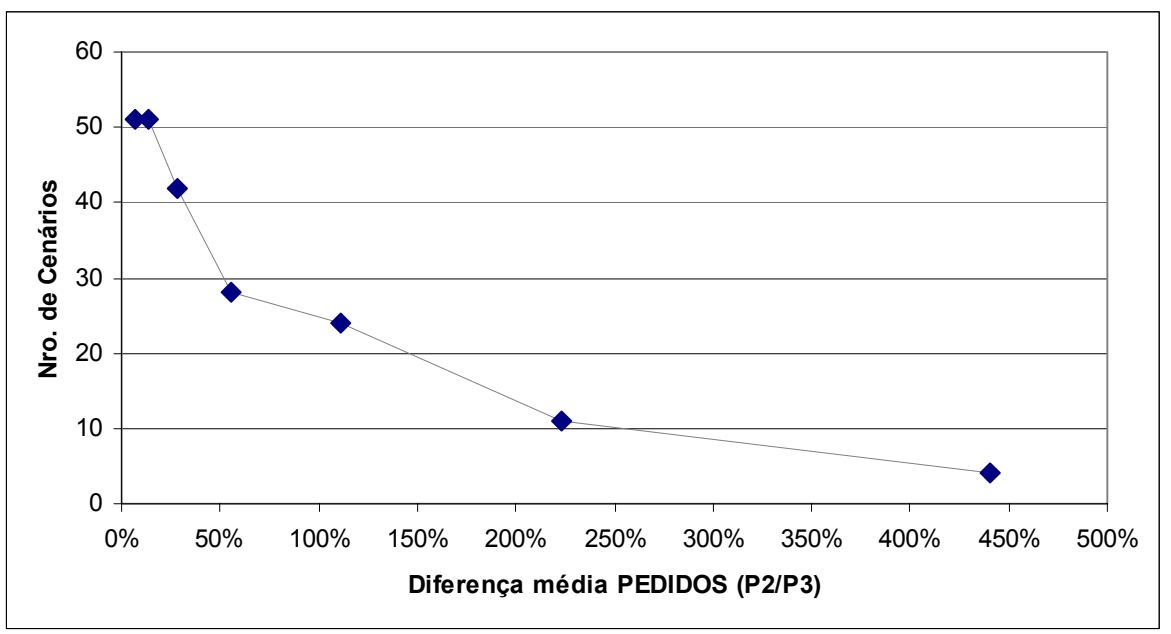

Gráfico 3: Diferenças \% do tamanho dos pedidos (P2/P3)

Além disso, as simulações comprovam que a política P2 gera aumentos desnecessários no tamanho médio dos pedidos, conforme referido na seção 5 (Tabela 1). Este fato faz com que o total de unidades solicitadas, ao longo do tempo, seja muito superior às reais necessidades expressas pela demanda. Conforme os resultados obtidos, os pedidos gerados pela política $\mathrm{P} 2$ são $12,5 \%$ maiores do que aqueles gerados pela política P3. O Gráfico 3 mostra que há aproximadamente 110 cenários com diferenças superiores a $25 \%$ (na montagem deste gráfico foram considerados somente valores maiores do que $5 \%$ ).

\section{CONCLUSÃO}

A escolha adequada da política de tratamento dos excessos de estoque é fundamental para o gerenciamento do EC. É possível demonstrar que há uma amplificação artificial do EC quando a política P1 é adotada, ou seja, quando o tamanho do pedido permanece inalterado, independentemente de seu valor ser positivo ou negativo. Quando a política P2 é adotada o tamanho médio dos pedidos é significativamente superior à demanda média, gerando estoques e custos desnecessários.

O modelo proposto por Chen et al. (2000), usado como referência nesta pesquisa, opera de modo satisfatório para determinados cenários, onde a relação entre lead time e número de períodos é favorável, isto é, quando o primeiro é menor do que o segundo. Nos casos em que a relação é desfavorável (lead time médio grande e número de períodos pequeno) ocorrem distorções no dimensionamento do EC, o que comprova a necessidade de desenvolvimento de modelos menos restritivos.

Os resultados obtidos através da comparação entre o modelo de Chen et al. (2000) e as simulações do EC evidenciam a importância da realização de pesquisas futuras sobre a eficácia das medidas propostas neste artigo, com vistas a um dimensionamento mais preciso deste fenômeno. A intensidade e o comportamento estocástico e serial do EC só podem ser adequadamente modelados se os excessos de estoque forem utilizados no cálculo do tamanho dos pedidos, através da adoção de uma política adequada de tratamento desses excessos.

Conforme apresentado no Gráfico 2, o EC pode aumentar indevidamente em torno de 20 vezes caso seja adotada uma política inadequada de tratamento dos excessos de estoque identificados em cada nível da cadeia de abastecimento. Em razão da natureza deste fenômeno, as simplificações presentes nos modelos de Lee et al. (1997), Chen et al. (2000) e Warburton (2004) geram distorções que, em função de sua magnitude, não devem ser desconsideradas.

\section{REFERÊNCIAS BIBLIOGRÁFICAS}

Anderson JR., E. G.; Fine, C. H.; Gilboy, G. J.; Parker, G. G. (2000) Upstream volatility in the supply chain: the machine tool industry as a case study. Production and Operations Management, v. 9, n. 3, p. 239-261.

Baganha, M. P. e Cohen, M. A. (1998) The stabilizing effect of inventory in supply chains. Operations Research, v. 46, n. 3, p. S72-S83.

Cachon, G. P. (1999) Managing supply chain demand variability with scheduled ordering policies. Management Science, v. 45, n. 6, p. 843-856.

Carlsson, C. e R. Fullér (2000) A fuzzy approach to the bullwhip effect. In: Cybernetics and Systems '2000, Proceedings of the Fifteenth European Meeting on Cybernetics and Systems research, Vienna, April 25-28, p. 228-233. Austrian Society for Cybernetic Studies.

Chen, F. Y. e Disney, S. M. (2003) The Order-up-to policy sweet spot using proportional controllers to eliminate the bullwhip problem. In EUROMA POMS Conference, Lago di Como, Itália, 16-18 June, p. 551-560.

Chen, F.; Z. Drezner; J. K. Ryan e D. Simchi-Levi (2000) Quantifying the Bullwhip Effect in a Simple Supply Chain: The Impact of Forecasting, Lead Times, and Information. Management Science, v. 46, n. 3, p. 436-443.

(2003) The Bullwhip Effect: managerial insights on the impact of forecasting and information on variability in a supply chain, In Quantitative models for supply chain management. 6.ed. Boston: Kluwer Academic Publishers, 885 p.

Croson, R. e Donohue, K. (2002) Experimental economics and supply chain management: experiments based on the Beer Distribution Game reveal manager's cognitive limitations. Interfaces, v. 32, n. 5, p. 74-82. 
(2003) Impact of POS data sharing on supply chain management: an experimental study. Production and Operations Management. v. 12 , n. 1, p. $1-11$.

Derrick, R. D. (2003) Challenging the bullwhip effect with advanced information sharing. Accenture Outlook, p. 1-2.

Donovan, M. (2003) Supply chain management: cracking the bullwhip effect. Working Paper. Disponível em www.rmdonovan.com.

Forrester, J. (1961) Industrial Dynamics. MIT Press and John Wiley \& Sons, New York.

Fransoo, J. C. e M. J. F. Wouters (2000) Measuring the Bullwhip Effect in the Supply Chain. Supply Chain Management, v. 5, n. 2, p. 78-89.

Hosoda, T. e Disney, S. M. (2004) An analysis of a three echelon supply chain model with minimum mean squared error forecasting. Second World Conference on POM and $15^{\text {th }}$ Annual POM Conference, Cancún: México, p. 1-24.

Kahn, J. (1987) Inventories and the volatility of production. American Economic Review. n. 77, p. 667-679.

Kunková, M. Optimization Methods and Bullwhip Effect, 2002. Disponível

http://www.fhi.sk/files/katedry/kove/ssov/VKOXI/.\%5CKuncova. pdf, último acesso em 22/12/2007.

Lee, H. L. e Billington, C. (1992) Managing supply chain inventory: pitfalls and opportunities. Sloan Management Review, n. 99, p. 6573.

Lee, H.; V. Padmanabhan e S. Whang (1997) Information Distortion in a Supply Chain: The Bullwhip Effect. Management Science, v. 43, n. 4 , p. $546-558$

Moyaux, T. (2000) Approche multi-agent de la gestion des chaînes logistiques, Doctoral Thesis, Université Laval, Canadá. Disponível em http://damas.ift.ulaval.ca, último acesso em 20/12/2007.

Moyaux, T.; Chaib-Draa, B.; D'amours, S. (2003) Agent-based simulation of the amplification of demand variability in a supply chain. Proceedings 4th Workshop on Agent-Based Simulation J.-P.Müller, M.-M. Seidel.

Sancar, A. Ü. (2003) Quantification of the bullwhip effect. Working Paper, Bogaziçi University, p. 1-9.

Warburton, R. D. H. (2004) An analytical Investigation of the Bullwhip Effect. Production and Operations Management, v. 13, n. 2, p. 150-160.

Watson, N. e Zheng, Y-S. (2001) Adverse effects of over-reaction to demand changes and improper forecasting. Working Paper, University of Pennsylvania. 